\title{
Circular RNA expression profile in blood according to ischemic stroke etiology
}

\author{
Aiora Ostolaza ${ }^{1 \dagger}$, Idoia Blanco-Luquin ${ }^{2^{*}}$, Amaya Urdánoz-Casado², Idoya Rubio ${ }^{1}$, Alberto Labarga4, \\ Beatriz Zandio ${ }^{1,3}$, Miren Roldán², Judith Martínez-Cascales², Sergio Mayor ${ }^{1,3}$, María Herrera ${ }^{1,3}$, Nuria Aymerich ${ }^{1,3}$, \\ Jaime Gallego ${ }^{1,3}$, Roberto Muñoz ${ }^{1,3+}$ and Maite Mendioroz ${ }^{1,2+}$
}

\begin{abstract}
Background: The discovery of novel biomarkers of stroke etiology would be most helpful in management of acute ischemic stroke patients. Recently, circular RNAs (circRNAs) have been proposed as candidate biomarkers of neurological conditions due to its high stability. circRNAs function as sponges, sequestering miRNAs and are involved in most relevant biological functions. Our aim was to identify differentially expressed circRNAs in acute ischemic stroke patients according to stroke etiology.
\end{abstract}

Methods: A comprehensive expression profile of blood circRNAs was conducted by Arraystar Human circRNA arrays (13,617 probes) on a discovery cohort of 30 stroke patients with different stroke etiologies by TOAST classification. Real-time quantitative PCR (RT-qPCR) was used to validate array results in a cohort of 50 stroke patients. Functional in silico analysis was performed to identify potential interactions with microRNAs (miRNAs) and pathways underlying deregulated circRNAs.

Results: A set of 60 circRNAs were found to be upregulated in atherotrombotic versus cardioembolic strokes (foldchange $>=1.5$ and $p$-value $\leq 0.05$ ). Differential expression of hsa_circRNA_102488, originated from UBA52 gene, was replicated in the validation cohort. RNA-binding proteins (RBPs) sites of hsa_circRNA_102488 clustered around AGO2 and FUS proteins. Further functional analysis revealed interactions between deregulated circRNAs and a set of miRNAs involved in stroke-related pathways, such as fatty acid biogenesis or lysine degradation.

Conclusion: Different stroke subtypes show specific profiles of circRNAs expression. circRNAs may serve as a new source of biomarkers of stroke etiology in acute ischemic stroke patients.

Keywords: Stroke, Etiology, circRNA, miRNA, Biomarker, UBA52

\section{Introduction}

Stroke is a common vascular disease that causes death and disability and is therefore a major challenge to current healthcare systems [1]. Nowadays, stroke diagnosis

\footnotetext{
*Correspondence: idoia.blanco.luquin@gmail.com

${ }^{\dagger}$ Aiora Ostolaza, Idoia Blanco-Luquin, Roberto Muñoz and Maite Mendioroz contribute equally to this work

${ }^{2}$ Neuroepigenetics Laboratory-Navarrabiomed-IdiSNA, Complejo Hospitalario de Navarra, Universidad Pública de Navarra (UPNA), IdiSNA (Navarra Institute for Health Research), C/Irunlarrea, 3, 31008 Pamplona, Navarra, Spain

Full list of author information is available at the end of the article
}

is still based on clinical criteria and imaging data, so clinicians are able to identify the etiology in about $75-80 \%$ of stroke cases by following the TOAST classification system [2]. In the remaining $20-25 \%$ of cases, the exact cause is unknown. It has been estimated that in about $25-30 \%$ of these cause-undetermined events, the underlying source of stroke could be paroxysmal atrial fibrillation (PAF) [1]. However, demonstrating this or other causes behind undetermined stroke events still remains a challenge in the clinical setting. Therefore, there is an increasing need for biomarkers capable of identifying stroke etiology in clinical practice. In recent years,

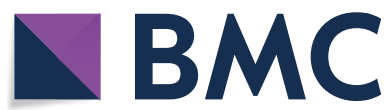

(c) The Author(s) 2020. This article is licensed under a Creative Commons Attribution 4.0 International License, which permits use, sharing, adaptation, distribution and reproduction in any medium or format, as long as you give appropriate credit to the original author(s) and the source, provide a link to the Creative Commons licence, and indicate if changes were made. The images or other third party material in this article are included in the article's Creative Commons licence, unless indicated otherwise in a credit line to the material. If material is not included in the article's Creative Commons licence and your intended use is not permitted by statutory regulation or exceeds the permitted use, you will need to obtain permission directly from the copyright holder. To view a copy of this licence, visit http://creativeco mmons.org/licenses/by/4.0/. The Creative Commons Public Domain Dedication waiver (http://creativecommons.org/publicdomain/ zero/1.0/) applies to the data made available in this article, unless otherwise stated in a credit line to the data. 
numerous studies have investigated a large amount of new blood biomarkers in relation with stroke etiology [3-7]. However, low sensitivity and specificity of the target biomarkers difficult their translation into clinical practice. Thus, the discovery of new molecules to aid the diagnosis of stroke etiology would be most helpful in this scenario.

By far, proteins represent the most widely studied class of molecules in the identification of biomarkers for cerebrovascular diseases. However, RNA molecules were also suggested as candidate stroke biomarkers about a decade ago $[8,9]$. In particular, brain and blood profiling in rat models revealed differential expression of certain microRNAs (miRNAs) after transient focal ischemia [8] and blood miRNAs were proposed as diagnostic and prognostic biomarkers in acute stroke patients [9].

Since then, a wide range of RNA species have been discovered, including circular RNAs (circRNAs). circRNAs are endogenous single-stranded RNA molecules which form a circle through a covalent binding [10]. These molecules are evolutionary conserved and very abundant in the human transcriptome [11]. circRNAs display a wide range of regulatory functions in RNA biology and gene expression. For instance, some circRNAs function as sponges that sequester miRNAs or RNA-binding proteins, regulating the expression of target genes [12]. In addition, given that circRNAs do not have free ends, they are resistant to endonuclease activity and therefore more stable than other linear RNA species such as messenger RNA (mRNA) or even microRNAs (miRNAs) [13]. This features make circRNAs promising biomarkers in different medical conditions. Indeed, circRNAs have recently been proposed as potential clinical biomarkers for neurological disorders [14]. However, comprehensive circRNAs expression has not been examined in stroke patients so far.

In this study, we have comprehensively profiled circRNAs in the peripheral blood of ischemic stroke patients during the acute stage. To this end, we used Arraystar Human circRNA Array $(8 \times 15 \mathrm{~K}$, Arraystar $)$, which surveys up to 13,617 probes to identify human circRNAs, in 30 acute stroke patients with different etiologies by TOAST classification [2]. As a result, we show that distinct circRNAs are differentially expressed in atherotrombotic versus cardioembolic stroke patients, arising as promising candidate biomarkers of stroke etiology.

\section{Methods}

\section{Study population}

Patients with acute ischemic stroke admitted to the emergency department of the Hospital of Navarra within the first $4.5 \mathrm{~h}$ after symptoms onset were included in the study. Among a cohort of 700 consecutive patients recruited from January 2015 to December 2016, 30 patients were included in the discovery cohort (Table 1) and 50 patients were included in the validation cohort (Additional file 2: Table S1). All participants completely fulfilled the etiology testing protocol (see methods below). Informed consent was obtained from all participants and the study was approved by the local Ethics Committee.

Table 1 Demographic and clinical characteristics of patients

\begin{tabular}{|c|c|c|c|c|}
\hline & $\begin{array}{l}\text { Atherothrombotic } \\
(n=8)\end{array}$ & Cardioembolic $(n=14)$ & Undetermined $(n=8)$ & p-value \\
\hline Age-years, median (IQR) & $70(55-80)$ & $75(70.5-77)$ & $66.5(49-77)$ & 0.366 \\
\hline Male, n (\%) & $7(87.5)$ & $7(50)$ & $5(62.5)$ & 0.214 \\
\hline High blood pressure, n (\%) & $6(75)$ & $12(85.7)$ & $3(37.5)$ & 0.056 \\
\hline Diabetes mellitus, n (\%) & $2(25)$ & $2(14.3)$ & $2(25)$ & 0.765 \\
\hline Dyslipidemia, n (\%) & $3(37.5)$ & $9(64.3)$ & $5(62.5)$ & 0.441 \\
\hline Smoker, n (\%) & $4(50)$ & $2(20)$ & $3(42.9)$ & 0.38 \\
\hline Cardiopathy, n (\%) & $3(37.5)$ & $6(42.9)$ & $0(0)$ & 0.093 \\
\hline Atrial fibrillation, n (\%) & $0(0)$ & $15(100)$ & $0(0)$ & $<0.001^{* * *}$ \\
\hline Peripheral arteropathy, n (\%) & $2(25)$ & $0(0)$ & $0(0)$ & 0.053 \\
\hline Basal mRankin, median (IQR) & $0.5(0-1)$ & $0(0-1.25)$ & $0(0-0.75)$ & 0.565 \\
\hline Basal NIHSS, median (RIQ) & $8.5(5-18)$ & $20(17-22)$ & $19(18-20)$ & $0.049^{*}$ \\
\hline Significant ipsilateral carotid stenosis (\%) & $8(100)$ & $0(0)$ & $1(14.3)$ & $<0.001^{* * *}$ \\
\hline Hemorrhagic transformation, n (\%) & $5(62.5)$ & $4(28.6)$ & $2(25)$ & 0.206 \\
\hline Discharge mRankin, median (IQR) & $4.5(2-6)$ & $4(2-5)$ & $3(0.5-5)$ & 0.434 \\
\hline
\end{tabular}

$I R Q$ interquartile range 


\section{Clinical, vascular and brain imaging protocol}

A detailed history of vascular risk factors including hypertension, atrial fibrillation, diabetes mellitus, dyslipidemia, tobacco, cardiovascular disease and peripheral atherosclerosis was recorded for each patient. In order to determine stroke etiology, a set of tests was performed including electrocardiogram (EKG), chest radiography, complete blood count, blood biochemistry analysis, carotid ultrasonography, transcranial doppler (TCD) examination, non-contrast cranial tomography (CT) at baseline, echocardiogram and $24 \mathrm{~h}$ holter monitoring. Patients were classified into etiological subgroups according to Trial of Org 10172 in Acute Stroke Treatment (TOAST) criteria [2].

\section{Microarray expression of circRNAs}

Venous blood samples were drawn from acute stroke patients within $24 \mathrm{~h}$ after admission. Total RNA was isolated from blood samples using miRNeasy Mini kit (QIAGEN, Redwood City, CA, USA) which enables purification of total RNA, including RNA from approximately 18 nucleotides upwards, following manufacturer's instructions. Genomic DNA was removed with a recombinant DNase (TURBO DNA-free ${ }^{\mathrm{TM}}$ Kit, Ambion, Inc., Austin, TX, USA). RNA integrity was assessed by electrophoresis on a denaturing agarose gel. Concentration of RNA was determined by OD260 using a NanoDrop ND-1000 spectrophotometer. Array star Human circRNA Array V2 analysis (Arraystar, Inc., Rockville, MD, USA) of the 30 selected stroke samples was performed as follows. Sample labeling and array hybridization were completed according to the manufacturer's protocol (Arraystar Inc.). Briefly, $2000 \mathrm{ng}$ of total RNAs were digested with RNase $R$ (Epicentre, Inc.) to remove linear RNAs and enrich circular RNAs. Then, the enriched circular RNAs were amplified and transcribed into fluorescent cRNA utilizing a random priming method (Arraystar Super RNA Labeling Kit; Arraystar). The labeled cRNAs were purified by RNeasy Mini Kit (Qiagen) and hybridized onto the Arraystar Human circRNA Array V2 $(8 \times 15 \mathrm{~K}$, Arraystar). After having washed the slides, the arrays were scanned by the Agilent Scanner G2505C (Agilent Technologies, Inc., Santa Clara, CA, USA).

\section{circRNAs microarray data}

Scanned images were imported into Agilent Feature Extraction software (version 11.0.1.1) for raw data extraction. Quantile normalization of raw data and subsequent data processing were performed using the $\mathrm{R}$ software ( $\mathrm{R}$ Project for Statistical Computing, Vienna, Austria) limma package. Normalized intensity values are shown in
Additional file 1: Fig. S1. After quantile normalization of the raw data, low intensity filtering was performed, and those circRNAs in which at least 8 out of 30 samples had flags in "P" or "M" ("All Targets Value") were retained for further analyses. Three distinct groups by stroke etiology (cardioembolic, atherotrombotic and undetermined) were analyzed by pairs, so three different comparisons were performed. When comparing two groups of profile differences (such as cardioembolic versus atherotrombotic), the fold change (FC) (i.e. the ratio of the group averages) between the groups for each circRNA was computed. Differentially expressed circRNAs between two groups were identified through FC filtering and statistical significance of the difference between two groups was estimated by Student's t-test. CircRNAs having FC $\geq 1.5$ and $p$-values $\leq 0.01$ were selected as significantly differentially expressed. Differentially expressed circRNAs with statistical significance between two groups were shown by Scatter plots and Volcano plots.

\section{Validation of array results by $\mathrm{qPCR}$}

Blood RNA isolated by miRNeasy Mini kit (QIAGEN, Redwood City, CA, USA) from the validation cohort $(\mathrm{n}=50)$ was used to validate array results. Complementary DNA (cDNA) was reverse transcribed from $500 \mathrm{ng}$ total RNA per sample with SuperScript ${ }^{\circledR}$ III First-Strand Synthesis Reverse Transcriptase (Invitrogen, Carlsbad, CA, USA) and primed with random primers. Real timeqPCR (RT-qPCR) reactions were performed in triplicate for each sample using Power SYBR ${ }^{\circledR}$ Green PCR Master Mix (Invitrogen, Carlsbad, CA, USA) in a QuantStudio 12 K Flex Real-Time PCR System (Applied Biosystems, Foster City, CA, USA). Divergent primer pairs were designed to overlap the backspliced junction by using Primer3 website (http://primer3.ut.ee/). Furthermore, GAPDH housekeeping gene and convergent primer pairs to amplify the host mRNA were designed by Real Time PCR tool (IDT, Coralville, IA, USA). Primer sequences are listed in Additional file 2: Table S2. At the designing stage, verification of primers specificity was carried out by PCR tool at the UCSC Genome Browser [15]. After amplification, we also checked that RT-qPCR reaction had generated a single-peak in the melting curve and a single amplicon of the correct size by performing agarose gel electrophoresis. The thermal cycling conditions consisted of an initial denaturation step at $95{ }^{\circ} \mathrm{C}$ for $10 \mathrm{~min}$ followed by 40 cycles of $15 \mathrm{~s}$ at $95^{\circ} \mathrm{C}$ and $1 \mathrm{~min}$ at $60{ }^{\circ} \mathrm{C}$. Expression levels of each corresponding linear transcript, GAPDH or convergent amplicon of the host gene, was used to normalize circRNA levels [16]. Relative expression level of circRNA in a particular sample was calculated by the delta delta-CT method, as previously described [17]. Non-template reactions were included as 
negative controls in each run. Finally, qPCR amplicons were subjected to Sanger sequencing [18] and checked for the presence of the predicted backspliced junctions in order to test their circularity.

\section{Functional in silico analysis}

Since certain circRNAs may function as sponges sequestering miRNAs and, therefore, may be involved in the regulation of gene expression, it was interesting to explore the potential interactions between differentially expressed circRNAs in stroke and miRNAs. The circRNA/microRNA interaction was predicted with Arraystar's home-made miRNA target prediction software based on TargetScan \& miRanda [19], and the differentially expressed circRNAs were annotated in detail with the circRNA/miRNA interaction information. Then, overrepresented miRNAs (those linked to at least four differentially expressed circRNAs) were analyzed by DIANA-mirPath v.3 software [20] to predict the underlying pathways.

CircInteractome tool provided a list of miRNAs potentially targeted by the circRNA of interest and mapped binding sites for RNA-binding proteins (RBPs) on it [21]. Next, DIANA-mirPath v.3 software [20] analyzed the pathways in which the outcome miRNAs were involved based on TarBase v7.0, microT-CDS v5.0 and TargetScan. Kyoto Encyclopedia of Genes and Genomes (KEGG) and Gene Ontology (GO) analyses were used to predict cell signaling pathways and functions related with the set of outcome miRNAs.

Moreover, in order to assess the interactions betweem the RBPs predicted to bind a particular differentially expressed circRNA, protein list was uploaded to the Search Tool for the Retrieval of Interacting Genes (STRING) tool (Version 11.0) [22] and filtered for interactions of high confidence (score $>0.7$ ) and PANTHER (Protein ANalysis THrough Evolutionary Relationships) Classification System (Version 14.1) [23]. Only those terms with a FDR-corrected $\mathrm{p}$-value $<0.05$ were reported.

\section{Statistical Analysis}

Statistical analysis was performed with SPSS 21.0 (IBM, Inc., USA). First, adjustment to normal distribution was tested for all continuous variables as per one-sample Kolgomorov-Smirnov test and the normal quantil-quantil (QQ) plots. Data represents the mean \pm standard deviation (SD) or the median (interquartile range). In the discovery cohort, univariate analysis of demographic and clinical characteristics was performed by ANOVA or Kruskall-Wallis test along with Chi square test. In the validation cohort, univariate analysis of demographic and clinical characteristics was performed by Student t-test or U Mann-Whitney test along with Chi square test. circRNA expression differences between two groups was estimated by Mann-Whitney U test. For all the comparisons, significance level was set at $\mathrm{p}$-value $<0.05$. SPSS 21.0 (IBM, Inc., USA) was used to draw graphs.

\section{Results \\ Differential expression of circRNAs in etiologic stroke subtypes}

To begin to ask whether circRNAs were differentially expressed in ischemic stroke depending on subtype etiology, we performed Arraystar Human circRNA Array V2 analysis, which included 13,617 distinct probes for human circRNAs, on a target group of 30 patients suffering from acute ischemic stroke with different etiologies according to TOAST classification [2], i.e. 14 cardioembolic, 8 atherotrombotic and 8 undetermined stroke. Demographic and clinical characteristics of patients included in the discovery cohort are shown in Table 1. Normalized intensity values showed similar distributions of the intensities (expression values) for all samples (Additional file 1: Fig. S1).

The main aim of this study was to identify differences in circRNA expression between atherotrombotic and cardioembolic stroke patients. We found 219 differentially expressed circRNAs $(\mathrm{FC} \geq 1.5$ and $\mathrm{p}$-value $\leq 0.05)$ in atherotrombotic versus cardioembolic stroke patients (Additional file 2: Tables S3 and S4). circRNA expression variation between the two compared groups is represented as volcano plots and scatter plots (Fig. 1a, Additional file 1: Fig. S2A). Despite we observed a higher number of downregulated (159) than upregulated (60) circRNAs in atherotrombotic compared to cardioembolic samples, the best findings in terms of statistical significance and magnitude of change were found among the upregulated circRNAs. Indeed, $11.7 \%$ (7) of upregulated circRNAs showed more than fourfold change in expression (Additional file 2: Table S3).

When comparing atherotrombotic versus undetermined strokes, 226 circRNAs were found to be differentially expressed $(\mathrm{FC} \geq 1.5$ and $\mathrm{p}$-value $\leq 0.05)$, being 87 circRNAs upregulated and 139 circRNAs downregulated in atherotrombotic compared to undetermined strokes (Additional file 2: Tables S5 and S6, Fig. 1b and Additional file 1: Fig. S2B). In this set, differences were not as strong as in the previous comparison of atherotrombotic versus cardioembolic strokes, and only one circRNA showed expression higher than fourfold change. Finally, a very few changes were found when comparing cardioembolic and undetermined strokes, as only 8 circRNAs were found to be upregulated and 9 circRNAs were downregulated in this comparison (Additional file 2: Table S7, Fig. 1c and Additional file 1: Fig. S2C). 

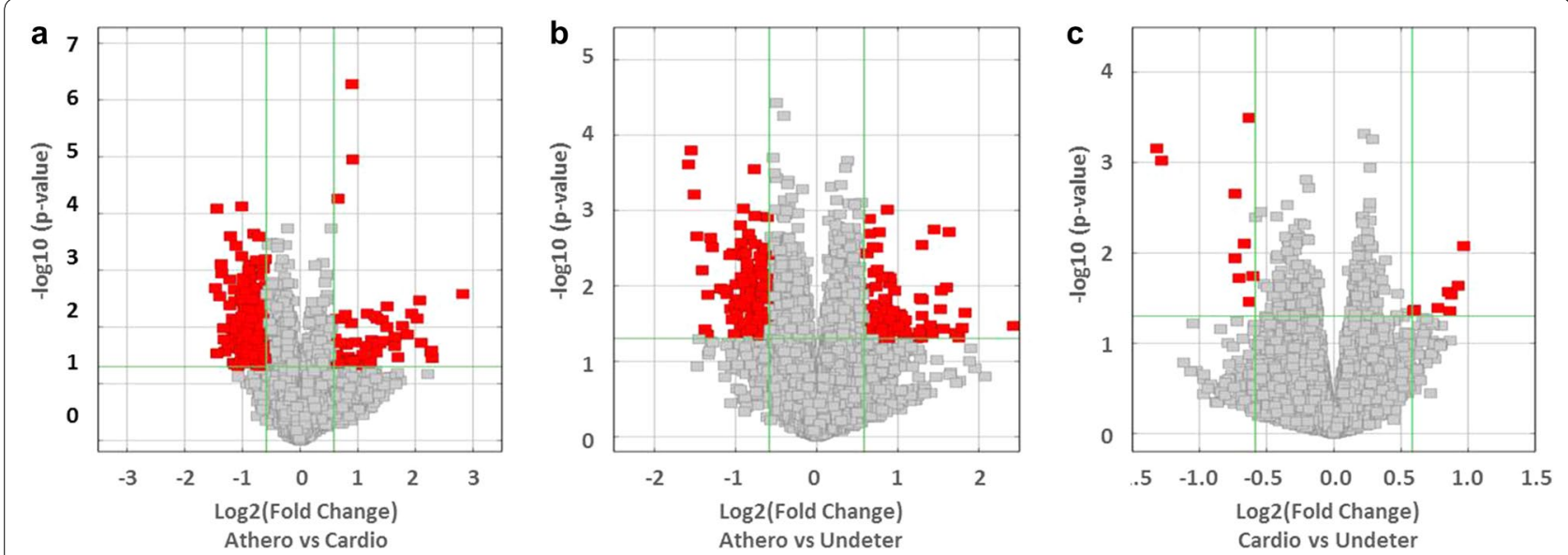

Fig. 1 Volcano-plots of differential expression of circRNAs for stroke etiology comparisons. Graphs visualize the relationship between fold-change (magnitude of change) in the $X$ axes and statistical significance (which takes both magnitude of change and variability into consideration) in the $Y$ axes. A high number of circRNAs are shown as differentially expressed between atherotrombotic versus cardioembolic strokes (a), and atherotrombotic versus undetermined strokes $(\mathbf{b})$ whereas cardioembolic versus undetermined strokes show less differences suggesting both groups are similar in terms of circRNA expression. The vertical green lines correspond to the threshold of 1.5 -fold up and down, respectively, and the horizontal green line represents a $p$-value $=0.05$. Red points in the plot represent the differentially expressed circRNAs with statistical significance which crossed the fold-change threshold

As shown in Fig. 2A, differentially expressed circRNAs seem to cluster in chromosomes 1, 2, 3 and 17 for the comparison atherotrombotic versus cardioembolic stroke (>15 hits); 1, 2, 7 and 17 for atherotrombotic versus undetermined ( $>15$ hits) and 4 and 17 for cardioembolic versus undetermined ( $>2.5$ hits). With these results in mind, circRNAs from chromosome 17, and even 1 and 2 , appear to be important for stroke etiology. In terms of distribution by chromosome regions, the number of differentially expressed circRNAs that are transcribed from protein-coding exons is the most abundant and comparable across downregulated and upregulated circRNAs (Fig. 2b). There were only a few intronic, antisense, sense overlapping and intergenic type circRNAs.

\section{Validation of differentially expressed circRNAs by RT-qPCR}

To validate the differences in circRNA expression, we focused on the atherotrombotic versus cardioembolic comparison since that was the main goal of our study. An expanded cohort which included samples previously analyzed in the microarray $(n=50 ; 25$ atherotrombotic and 25 cardioembolic samples) was used to this end. Next, 3 upregulated and 2 downregulated circRNAs in atherotrombotic versus cardioembolic stroke patients were selected based on highest fold change and/or lowest p-value prioritization (Table 2). Then, we performed RTqPCR to amplify and quantify the chosen circRNAs, their host mRNAs and GAPDH mRNA levels. Compared to the linear transcript, only hsa_circRNA_102488 showed a statistically significant change in expression between etiology subtypes. hsa_circRNA_102488 is originated from the ubiquitin a-52 residue ribosomal protein fusion product 1 (UBA52) gene which is located at chromosome 19 (Fig. 3a). Sanger sequencing confirmed the presence of the backsplicing junction between UBA52' exons 3 and 2 (Fig. 3b). Mann-Whitney U test revealed that expression levels of hsa_circRNA_102488 (alias hsa_circ_0005568) were lower for atherotrombotic compared to cardioembolic samples when normalizing to GAPDH mRNA (p-value $<0.01$ ) (Fig. 3c), as well as to the corresponding UBA52 mRNA (p-value <0.001) (Fig. 3d). Interestingly, we did not found significant differences in UBA52 mRNA expression levels between atherotrombotic and cardioembolic stroke samples (Fig. 3e).

\section{Functional in silico analysis of differentially expressed circRNAs}

In order to explore potential interactions between differentially expressed circRNAs in stroke subtypes and target miRNAs, a bioinformatics analysis was performed (see "Methods"). First, interactions between circRNAs and their target miRNAs were predicted by Arraystar's home-made software. Then, we selected the overrepresented miRNAs in the three comparisons (those miRNAs linked to at least four differentially expressed circRNAs), and characterized their related pathways by DIANA-mirPath ( $\mathrm{p}$-value threshold $\leq 0.05$ ). Overrepresented miRNAs were associated with fatty acid biosynthesis and metabolism, lysine degradation, arrhythmogenic right ventricular 


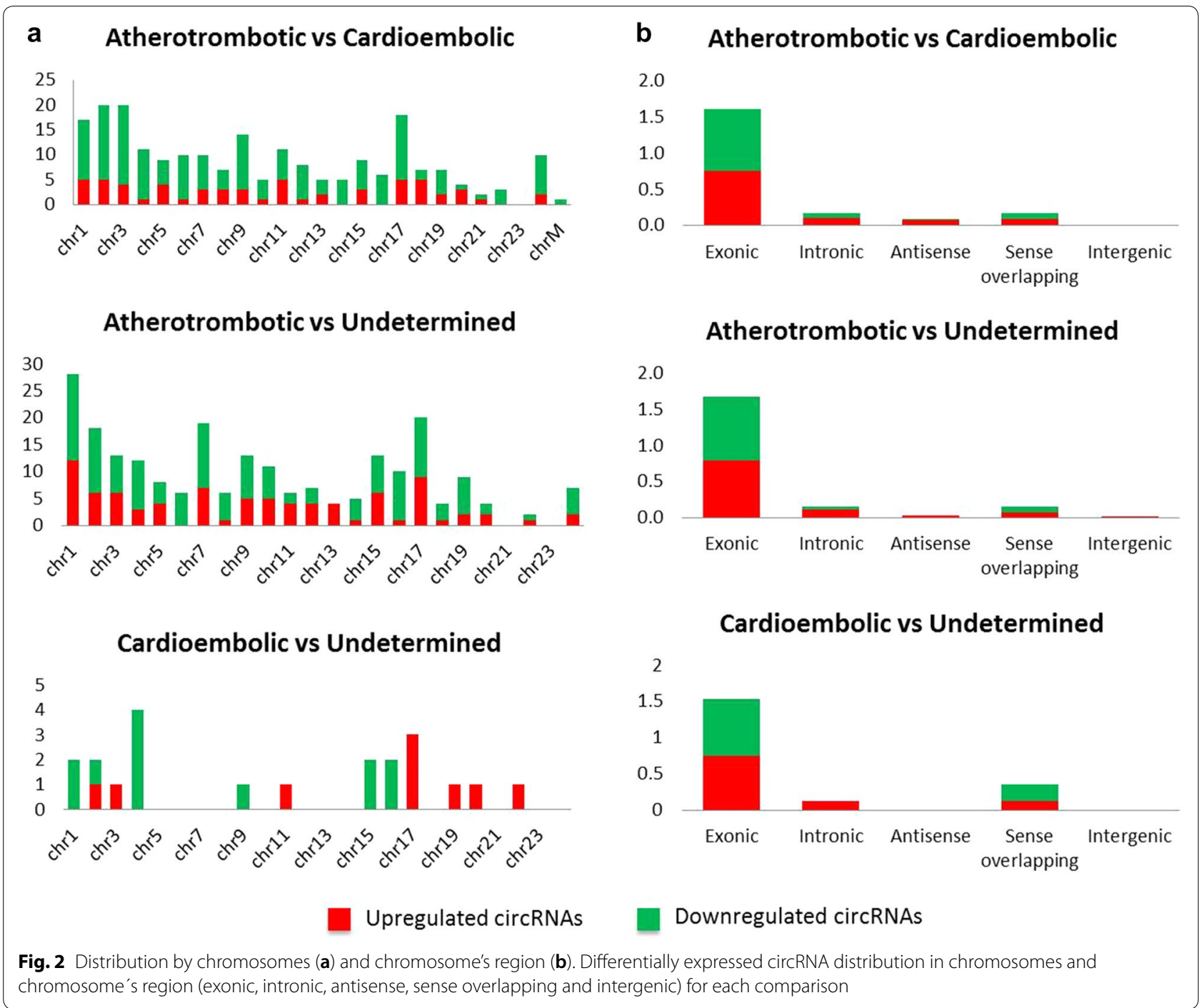

Table 2 Deregulated circRNA chosen for qPCR validation

\begin{tabular}{lllllllll}
\hline Regulation & CircRNA & CircRNA type & FC (abs) & P-value & Chromosome position & Gene symbol \\
\hline Up & hsa_circRNA_001359 & intronic & 7.0479384 & 0.002611197 & chr1 & 169663839 & 169664181 & SELL \\
& hsa_circRNA_103559 & exonic & 4.8769307 & 0.035206182 & chr3 & 196118683 & 196120490 & UBXN7 \\
\multirow{2}{*}{ Down } & hsa_circRNA_104220 & exonic & 4.8572966 & 0.026033314 & chr6 & 150092297 & 150094305 & PCMT1 \\
& hsa_circRNA_102488 & exonic & 2.7229035 & $8.17917 E-05$ & chr19 & 18684102 & 18684558 & UBA52 \\
& hsa_circRNA_104748 & exonic & 2.3292681 & 0.001448543 & chr9 & 20907148 & 20929595 & FOCAD \\
\hline
\end{tabular}

CircRNA deregulated circRNA with greater intensity values in atherotrombotic stroke patients compared with cardioembolic; circRNA_type: the circRNAs are classified into 5 types: "exonic", "intronic", "antisense", "sense overlapping" and "intergenic"; $F C$ absolute ratio (no log scale) of normalized intensities between two conditions; $p$-value $\mathrm{p}$-value calculated from unpaired t-test; Annotations, include chromosome position and Gene Symbol

cardiomyopathy (ARVC), adrenergic signaling in cardiomyocytes or hypertrophic cardiomyopathy (HCM) as shown by KEGG analysis (p-value threshold $<0.05$ ) (Additional file 1: Fig. S3A). Moreover, these miRNAs were linked to cellular nitrogen compound metabolic process, homophilic cell adhesion via plasma membrane adhesion molecules, cell adhesion, blood coagulation or neurotrophin tyrosine-kinase (TRK) receptor signaling pathway in $\mathrm{GO}$ analysis (p-value threshold <0.05) (Fig. 4a). 


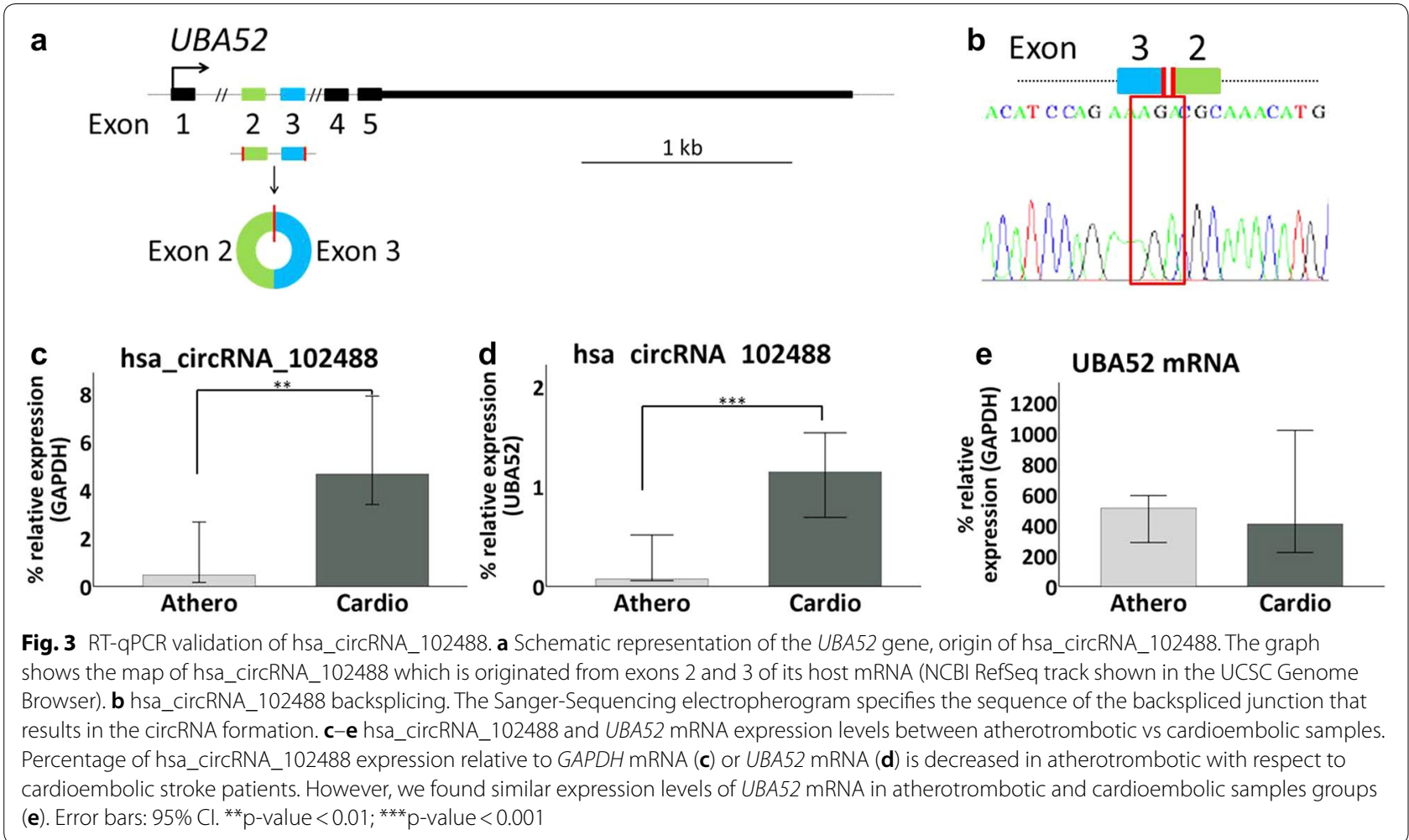

Regarding the validated circRNA, hsa_circRNA_102488, CircInteractome tool [21] showed one $7 \mathrm{mer}-\mathrm{m} 8$ or 7 mer-1a type target site shared by the following miRNAs: hsa-miR-1182, hsa-miR-1299, hsamiR-431, hsa-miR-516b, hsa-miR-668 and hsa-miR-766. DIANA-mirPath analysis revealed that this set of miRNAs converged on the pathways of fatty acid biosynthesis and metabolism, extracellular matrix (ECM)-receptor interaction, lysine degradation or arrhythmogenic right ventricular cardiomyopathy (ARVC) as shown by KEGG analysis ( $p$-value threshold $<0.001$ (Additional file 1: Fig. S3B, C). They seemed to have a common function as RNA-, ion- and enzyme-binding molecules, a protein and nucleic acid binding transcription factor activity and to be involved in cellular nitrogen compound metabolic, biosynthetic, cellular protein modification and gene expression processes ( $\mathrm{GO}$ analysis, p-value threshold <0.05) (Fig. 4b, c).

In addition, up to 10 RBPs sites matching to hsa_circRNA_102488 were identified by CircInteractome: 3 for SFRS1, 2 for AGO2, HuR, IGF2BP2 and PTB and 1 for CAPRIN1, DGCR8, FMRP, IGF2BP1 and LIN28B. STRING clustered these RBPs around AGO2 (proteinprotein interaction, $\mathrm{PPI}$, enrichment $\mathrm{p}$-value $<1.0 \mathrm{e}-16$ ) (Additional file 1: Fig. S4A-C) and PANTHER related them to two categories of molecular functions: RNA binding and catalytic activity. Moreover, other RBPs sites that matched to the hsa_circRNA_102488 flanking regions were identify for EIF4A3 (9 Tags), HNRNPC, $H u R, U 2 A F 65$ and FUS genes. After functional analysis, we observed that these RBPs clustered around FUS (PPI enrichment $\mathrm{p}$-value $<2.11 \mathrm{e}-10$ ) and related to catalytic activity, IGF2BP1 and IGF2BP3 to binding molecular function and ZC3H7B separately but also to catalytic activity function (STRING and PANTHER tools) (Additional file 1: Fig. S4D-F).

A diagram illustrating impact pathways mentioned above is shown in Fig. 5.

\section{Discussion}

In this study, we identified circRNAs that were differentially expressed in human blood according to stroke etiology. The strongest differences were found for the comparison between atherotrombotic and cardioembolic stroke. Differentially expressed circRNAs were predicted to predominantly interact with a set of miRNAs involved in stroke-related pathways, including fatty acid biosynthesis, lysine degradation, arrhythmogenic right ventricular cardiomyopathy (ARVC) or hypertrophic cardiomyopathy (HCM), among others.

circRNAs are a new class of non-coding RNA molecules with circular morphology due to the formation of a covalent junction between the $3^{\prime}$ and $5^{\prime}$ ends. These molecules are generated from a parental (also known as 


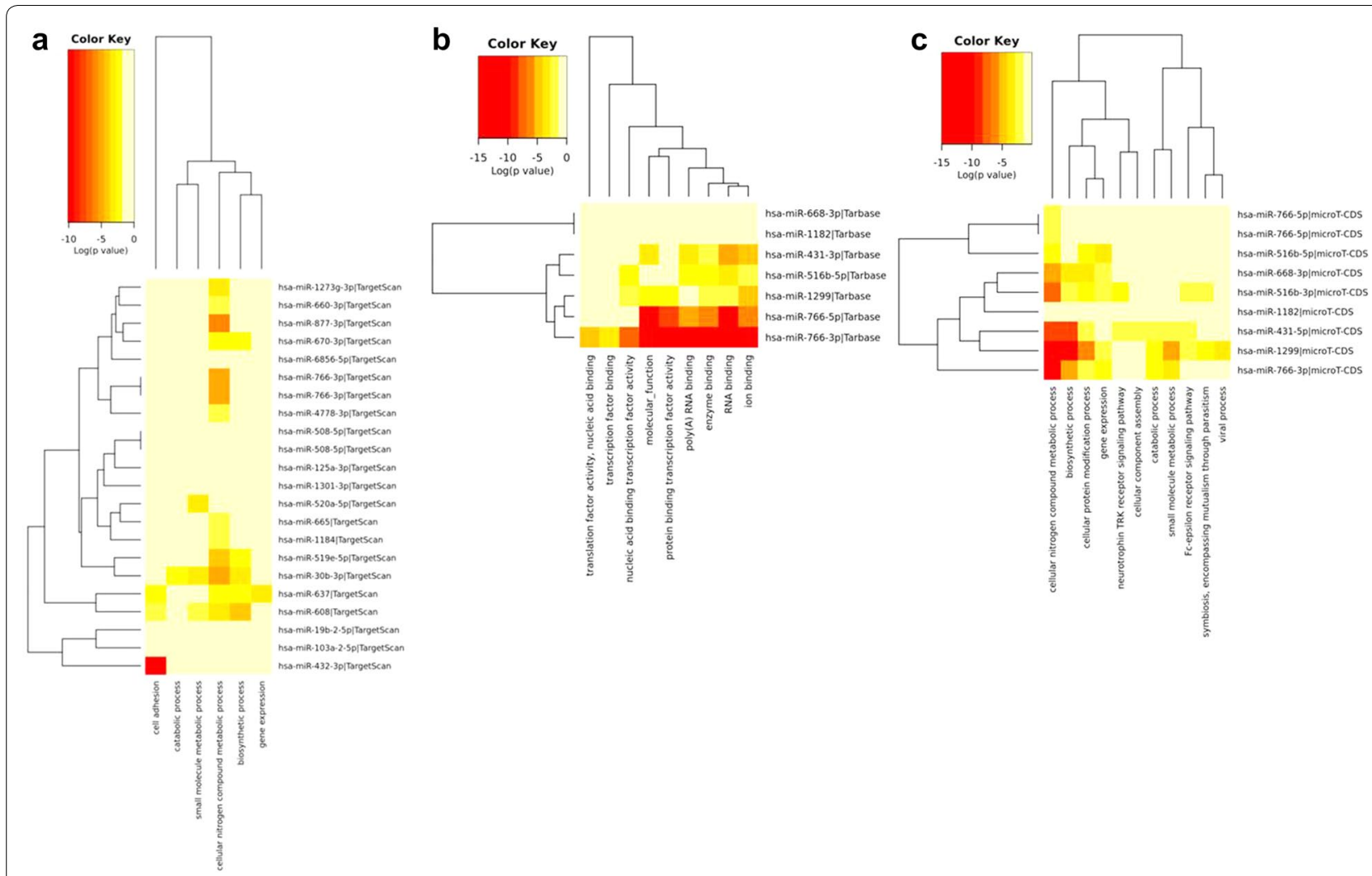

Fig. 4 GO analysis by mirPath v.3. The image shows the heatmaps corresponding to the analysis performed with the overrepresented miRNAs in the three comparisons (atherotrombotic-cardioembolic-undetermined) by TargetScan (a) and the analysis involving the target miRNAs for hsa_ circRNA_102488 in accordance with molecular function (b, by Tarbase) or biological process (c, by microT-CDS)

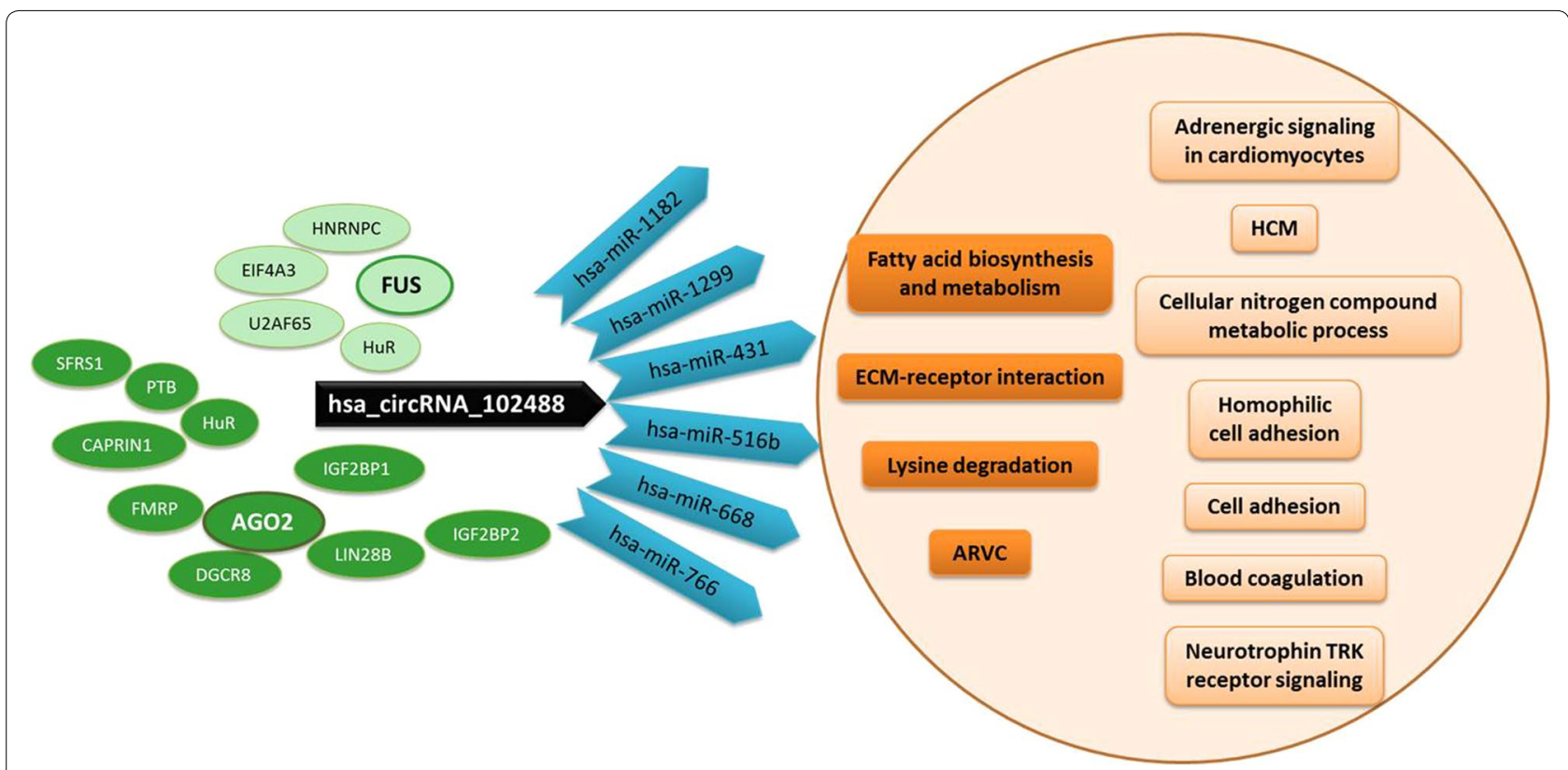

Fig. 5 hsa_circRNA_102488 impact pathways. The cartoon shows miRNAs (in blue) sharing a target site with hsa_circRNA_102488 (in black) and the pathways in which they converge (in dark orange), within those associated with the overrepresented miRNAs in the three comparisons (in light orange). RBPs sites matching to hsa_circRNA_102488 clustered around AGO2 (in dark green) and RBPs sites that matched to its flanking regions clustered around FUS (in light green). ARVC: Arrhythmogenic Right Ventricular Cardiomyopathy; HCM: Hypertrophic Cardiomyopathy 
host) pre-messenger RNA through a particular alternative splicing process known as backsplicing $[24,25]$. The covalent bond confers resistance to ribonucleases preventing degradation and making circRNAs very stable molecules. Furthermore, circRNAs are expressed in most mammalian tissues, particularly in the brain and have been also detected in the human blood [26-28]. All these characteristics make circRNAs potential biomarkers of human diseases and, especially, of neurological diseases.

Indeed, previous reports suggesting the potential role of circRNAs as biomarkers or therapeutic targets in neurological conditions, such as multiple sclerosis or epilepsy, have been already published [29-31]. In the stroke field, a number of changes in circRNAs expression levels have been recently described. In 2017, it was observed for the first time that circRNAs are altered in the mouse brain after transient focal ischemia [32, 33]. Despite these initial observations, as far as we know, a comprehensive profiling of circRNAs in human stroke or its relationship to stroke etiology has not previously been reported.

In our study, a set of 219 differentially expressed circRNAs between atherotrombotic and cardioembolic stroke was found in the blood of acute stroke patients. The most robust statistical differences were observed among the 60 circRNAs up-regulated in atherotrombotic compared to cardioembolic samples. Most of them tended to cluster in chromosome 17 and were of exonic origin, which is not surprising since the majority of known circRNA molecules are exonic and therefore, there is an overrepresentation of exonic circRNAs in the Arraystar microarray.

Even though a large number of circRNAs were shown to be differentially expressed in atherotrombotic versus undetermined stroke samples, there were hardly any differences between cardioembolic and undetermined strokes. This result suggests that the undetermined stroke group may harbor a great proportion of nonrecognized cardioembolic strokes, which is in line with current knowledge in the clinical setting. In fact, it has been described that some patients with stroke of undetermined etiology may have atrial structural or functional changes thus increasing the risk of cardioembolism [34].

In recent years, functions of circRNAs are beginning to be understood. It has been observed that circRNAs can act at different levels of gene regulation, including transcription, mRNA splicing or translation. However, the best known function so far is the regulation of gene expression by acting as miRNA sponges: circRNAs have binding sites for specific miRNAs and act by "sequestering" them to prevent miRNAs from exerting translational repression. Thus far, a few stroke-related circRNAs acting as miRNA sponges have been observed. circRNA DLGAP4 functions as a sponge for miRNA-143 and therefore, inhibits miRNA-143 activity. Interestingly, overexpression of circRNA DLGAP4 ameliorates neurological deficit and infarct volume after transient focal ischemia in a stroke mouse model. circRNA DLGAP4 seems to significantly attenuate blood-brain barrier damage by regulating tight junction protein expression and endothelial-mesenchymal transition in endothelial cells [35]. Other example of deregulated circRNAs in stroke is circRNA Hectd1, which was found to be upregulated in the brain of mice after transient middle cerebral artery occlusion (tMCAO). circRNA HECTD1 participates in the inhibition of astrocyte activation via macroautophagy/autophagy by acting as a sponge for MIR-142 [36]. Interestingly, circRNA HECTD1 was increased in peripheral blood mononuclear cells from acute ischemic stroke patients compared to controls and higher levels of circRNA HECTD1 were related with stroke recurrence [37]. The circRNA TLK1, which acts as an endogenous miR-335-3p sponge, has been proved to be detrimental in ischemic brain injury. It was increased in the mouse brain after tMCAO and knocking down circRNA TLK1 improve neurological deficits and infarct volume in this stroke mouse model. Remarkably, circRNA TLK1 was observed to be also elevated in human patients after ischemic stroke [38].

In the present study, differentially expressed circRNAs were predicted to interact with numerous miRNAs. Functional study showed that those miRNAs were involved in pathways associated with stroke etiology or pathogenesis, such as fatty acids biogenesis, lysine degradation or development of cardiopathy, including ARVC and HCM. Elevated levels of free fatty acids have been associated with cardioembolic etiology of stroke irrespective of the presence of atrial fibrillation $[39,40]$ and predict stroke recurrence in patients with atrial fibrillation [41]. Although the underlying mechanisms remain unclear, fatty acids are major components of epicardial fat and it is known that thickness of epicardial fat is related with the presence of atrial fibrillation [42]. According to that, serum fatty acid binding proteins are proposed as biomarkers for atrial fibrillation [43]. However, fatty acids seem independent predictors of stroke events so they may be directly related to thrombogenesis [39, 40].

Interestingly, lysine degradation pathway showed a strong association with overrepresented miRNAs in our study. Lysine is an essential aminoacid with pleiotropic functions in humans. In addition to proteinogenesis, lysine is involved in the crosslinking of collagen peptides, the uptake of calcium and iron and is the precursor of carnitine, which in turn is essential in the metabolism of fatty acids [44]. Lysine degradation differs in brain from that of in extracerebral tissues and it is known to be regulated by thyroid hormones [45]. For now, its role in stroke is not yet well understood. A recent study among 
hypertensive subjects revealed altered metabolic pathways, including lysine degradation, related to incident ischemic stroke [46]. Moreover, a metabolomic study showed that levels of serum lysine catabolites were low in patients at high-risk of suffering ischemic stroke [47].

Despite ARVC is not among the classical embolic sources of stroke events, ARVC pathways have been recently associated with ischemic stroke in a comprehensive long non-coding RNA transcriptomic study [48] and this is consistent with our results. HCM is a common hereditary cardiomyopathy frequently associated with sudden death. It has been described that atrial fibrillation with risk for progressive heart failure and embolic stroke occurs in $20 \%$ of patients with HCM [49].

In this study, we were able to validate hsa_circRNA_102488 in an expanded cohort of atherotrombotic versus cardioembolic strokes. This circRNA is formed from the pre-mRNA of UBA52 gene and has a 456 bp genomic length. hsa_circRNA_102488 was found to be highly expressed in brain tissues [27]. No previous records of the association between $U B A 52$ gene and stroke have been reported to our knowledge.

Functional analysis revealed that hsa_circRNA_102488 harbors a binding site for 6 different miRNAs. Intriguingly, this set of 6 miRNAs is enriched in pathways similar to those found for the overrepresented miRNAs, namely fatty acids biogenesis, lysine degradation and ARVC. This result suggests that hsa_circRNA_102488 may be representative of the altered circRNA network and its related pathways underlying stroke etiology and makes hsa_circRNA_102488 an interesting candidate worth to be explored in futures studies.

Among known functions, circRNAs interact with RNA binding proteins (RBPs) to influence gene transcription and translation [50]. In hsa_circRNA_102488, the analysis revealed RBPs binding sites that cluster around two RBPs: argonaute 2 (Ago2) and RNA-binding protein FUS. Argonaute 2 is a critical component of the RNA-induced silencing complex (RISC) and, as a consequence, a master regulator of miRNAs-dependent gene silencing pathway. Interestingly, it has been observed that Ago2 accumulates and undergoes hydroxylation following hypoxia, thus Ago2 emerges as a major regulator of the pathological responses to hypoxia [51]. In fact, stroke substantially modifies Ago2-associated miRNA profiles in the brain in a stroke rat model [52]. On the other hand, FUS participates in DNA repair mechanisms [53] and is frequently mutated in amyotrophic lateral sclerosis [54]. However, no previous works on the role of FUS in stroke has been previously reported.

The determination of circRNA as biomarkers in the etiology of stroke would be a great advance in the diagnostic process of cerebral vascular diseases. It should be noted that circRNAs are especially interesting as biomarkers because they represent a gene regulatory mechanism for several genes at once and, despite being RNA molecules, they are extremely stable. A large part of the study carried out on these patients is aimed to find the cause of the stroke, and in up to $25 \%$ of cases a clear etiology cannot be established. The risk factors that predispose to presenting an atherothrombotic stroke are widely known and current guidelines make general recommendations to prevent them. However, patients who remain without a specific etiological diagnosis would not benefit from these guidelines. Moreover, knowing the cause of stroke in a rapid and non-invasive way would help to improve secondary prevention and avoid unnecessary studies.

As a discovery study, where typically a large number of molecules are measured in a reduced number of subjects, an obvious limitation of this study is the limited sample size. Therefore, we should be cautious with our conclusions. For the sake of external validity, these findings should be replicated in blood samples of an independent cohort of ischemic stroke patients with different etiologies.

\section{Conclusions}

The present study showed a set of differentially expressed circRNAs in peripheral blood from different stroke etiologic subtypes. These circRNAs revealed interesting pathways underlying stroke etiology, such as fatty acid biogenesis or lysine degradation. Due to their molecular features, circRNAs may be useful as candidate biomarkers for stroke etiology. Large and independent cohort studies will be needed to further investigate the role of circRNAs as stroke biomarkers.

\section{Supplementary information}

Supplementary information accompanies this paper at https://doi. org/10.1186/s13578-020-00394-3.

Additional file 1. Additional figures.

Additional file 2. Additional tables.

\begin{abstract}
Acknowledgements
We want to kindly thank technical staff from Neuroradiology Angiography Room and the Stroke Unit Team and of the Complejo Hospitalario de Navarra, Spain, for the support to collect blood samples. We also want to thank personnel of the Navarrabiomed Biobank, including Valle Coca, Leticia San José, Ana Purroy and Isabel Gil MD, PhD, for their support. We are very grateful to David Otaegui, PhD from Biodonostia Institute, and his PhD student Leire Iparraguirre, for their technical and scientific support. Finally, we are very grateful to the patients that generously donor samples for this study.
\end{abstract}

\section{Authors' contributions}

$\mathrm{AO}$ and IBL contributed to running experiments, acquisition, analysis and interpretation of data, and drafting/revising the manuscript for content. AU contributed to analysis of data, figure drawing and drafting/revising the manuscript for content. IR contributed to revising subject diagnosis, classification of patients and drafting/revising the manuscript for content. $\mathrm{AL}$ 
contributed to bioinformatics analysis and drafting the manuscript. BZ contributed to analyze results and drafting/revising the manuscript for content. $M R$ and JMC contributed to running experiments and data collection. SM, MH, NA and JG contributed to analyze results and drafting/revising the manuscript for content. RM and MM contributed to study concept and design, statistical analysis, analysis and interpretation of data, drafting/revising the manuscript for content, study supervision and obtaining funding. All authors read and approved the final manuscript.

\section{Funding}

This work was supported by Navarre Government Funding (Industry and Health department) through ADITECH and by RED INVICTUS (RD16/0019/0024) from the Institute of Health Carlos III, jointly funded by European Regional Development Fund (ERDF), European Union. AUC received a grant "Doctorados industriales 2018-2020" founded by Government of Navarra and MM received a grant "Programa de intensificación" founded by "LaCaixa Foundation" and Fundación Caja-Navarra.

\section{Availability of data and materials}

The datasets used and/or analysed during the current study are available from the corresponding author on reasonable request.

\section{Ethics approval and consent to participate}

All procedures performed in studies involving human participants were in accordance with the ethical standards of the institutional and/or national research committee (clinical research ethics committee of Navarre; Pyto 2015/21) and with the 1964 Helsinki declaration and its later amendments or comparable ethical standards.

\section{Informed consent}

Informed consent was obtained from all individual participants included in the study.

\section{Competing interest}

The authors declare that they have no competing interests.

\section{Author details}

${ }^{1}$ Department of Neurology, Complejo Hospitalario de Navarra-IdiSNA (Navarra Institute for Health Research), 31008 Pamplona, Navarra, Spain. ${ }^{2}$ Neuroepigenetics Laboratory-Navarrabiomed-IdiSNA, Complejo Hospitalario de Navarra, Universidad Pública de Navarra (UPNA), IdiSNA (Navarra Institute for Health Research), C/Irunlarrea, 3, 31008 Pamplona, Navarra, Spain. ${ }^{3}$ Stroke Unit, Department of Neurology, Complejo Hospitalario de Navarra- IdiSNA (Navarra Institute for Health Research), 31008 Pamplona, Navarra, Spain. ${ }^{4}$ Bioinformatics Unit, Navarrabiomed, Public University of Navarre (UPNA), IdiSNA (Navarra Institute for Health Research), C/Irunlarrea, 3, 31008 Pamplona, Navarra, Spain.

Received: 8 January 2020 Accepted: 24 February 2020

Published online: 10 March 2020

\section{References}

1. Szegedi I, Szapáry L, Csécsei P, Csanádi Z, Csiba L. Potential biological markers of atrial fibrillation: a chance to prevent cryptogenic stroke. Biomed Res Int. 2017;2017:8153024. https://doi.org/10.1155/2017/81530 24.

2. Adams HP, Bendixen BH, Kappelle $\sqcup$, Biller J, Love BB, Gordon DL, et al. Classification of subtype of acute ischemic stroke. Definitions for use in a multicenter clinical trial. TOAST. Trial of Org 10172 in acute stroke treatment. Stroke. 1993;24(1):35-41.

3. Yin R, Ma A, Pan X, Yang S. Biomarkers of cerebral microembolic signals. Clin Chim Acta. 2017;475:164-8. https://doi.org/10.1016/j. cca.2017.10.028.

4. Sun H, Zhao J, Zhong D, Li G. Potential serum biomarkers and metabonomic profiling of serum in ischemic stroke patients using UPLC/QTOF MS/MS. PLoS ONE. 2017;12(12):e0189009. https://doi.org/10.1371/ journal.pone.0189009.

5. Dagonnier M, Cooke IR, Faou P, Sidon TK, Dewey HM, Donnan GA, et al. Discovery and longitudinal evaluation of candidate biomarkers for ischaemic stroke by mass spectrometry-based proteomics. Biomark
Insights. 2017;12:1177271917749216. https://doi.org/10.1177/11772 71917749216.

6. Llombart V, Garcia-Berrocoso T, Bustamante A, Fernandez-Cadenas I, Montaner J. Cardioembolic stroke diagnosis using blood biomarkers. Curr Cardiol Rev. 2013;9(4):340-52.

7. Jauch EC, Barreto AD, Broderick JP, Char DM, Cucchiara BL, Devlin TG, et al. Biomarkers of acute stroke etiology (BASE) study methodology. Transl Stroke Res. 2017. https://doi.org/10.1007/s12975-017-0537-3.

8. Jeyaseelan K, Lim KY, Armugam A. MicroRNA expression in the blood and brain of rats subjected to transient focal ischemia by middle cerebral artery occlusion. Stroke. 2008;39(3):959-66. https://doi.org/10.1161/strok eaha.107.500736.

9. Tan KS, Armugam A, Sepramaniam S, Lim KY, Setyowati KD, Wang CW, et al. Expression profile of MicroRNAs in young stroke patients. PLoS ONE. 2009;4(11):e7689. https://doi.org/10.1371/journal.pone.0007689.

10. Chen LL, Yang L. Regulation of circRNA biogenesis. RNA Biol. 2015;12(4):381-8. https://doi.org/10.1080/15476286.2015.1020271.

11. Salzman J, Gawad C, Wang PL, Lacayo N, Brown PO. Circular RNAs are the predominant transcript isoform from hundreds of human genes in diverse cell types. PLoS ONE. 2012;7(2):e30733. https://doi.org/10.1371/ journal.pone.0030733.

12. Chen L, Huang C, Wang X, Shan G. Circular RNAs in eukaryotic cells. Curr Genomics. 2015;16(5):312-8. https://doi.org/10.2174/138920291666615 0707161554.

13. Bolha L, Ravnik-Glavač M, Glavač D. Circular RNAs: biogenesis, function, and a role as possible cancer biomarkers. Int J Genomics. 2017;2017:6218353. https://doi.org/10.1155/2017/6218353.

14. Lu D, Xu AD. Mini review: circular RNAs as potential clinical biomarkers for disorders in the central nervous system. Front Genet. 2016;7:53. https:// doi.org/10.3389/fgene.2016.00053.

15. Kent WJ, Sugnet CW, Furey TS, Roskin KM, Pringle TH, Zahler AM, et al. The human genome browser at UCSC. Genome Res. 2002;12(6):996-1006. https://doi.org/10.1101/gr.229102.

16. Vandesompele J, De Preter K, Pattyn F, Poppe B, Van Roy N, De Paepe A, et al. Accurate normalization of real-time quantitative RT-PCR data by geometric averaging of multiple internal control genes. Genome Biol. 2002;3(7):34.

17. Livak KJ, Schmittgen TD. Analysis of relative gene expression data using real-time quantitative PCR and the 2(-Delta Delta C(T)) Method. Methods (San Diego, Calif). 2001;25(4):402-8. https://doi.org/10.1006/ meth.2001.1262.

18. Sanger F, Nicklen S, Coulson AR. DNA sequencing with chain-terminating inhibitors. Proc Natl Acad Sci USA. 1977;74(12):5463-7.

19. Pasquinelli AE. MicroRNAs and their targets: recognition, regulation and an emerging reciprocal relationship. Nat Rev Genet. 2012;13(4):271-82. https://doi.org/10.1038/nrg3162.

20. Vlachos IS, Zagganas K, Paraskevopoulou MD, Georgakilas G, Karagkouni D, Vergoulis T, et al. DIANA-miRPath v3.0: deciphering microRNA function with experimental support. Nucleic Acids Res. 2015;43(W1):W460-6. https://doi.org/10.1093/nar/gkv403.

21. Dudekula DB, Panda AC, Grammatikakis I, De S, Abdelmohsen K, Gorospe M. Circlnteractome: a web tool for exploring circular RNAs and their interacting proteins and microRNAs. RNA Biol. 2016;13(1):34-42. https:// doi.org/10.1080/15476286.2015.1128065.

22. Salwinski L, Miller CS, Smith AJ, Pettit FK, Bowie JU, Eisenberg D. The database of interacting proteins: 2004 update. Nucleic Acids Res. 2004;32(Database issue):D449-51. https://doi.org/10.1093/nar/gkh086.

23. Thomas PD, Campbell MJ, Kejariwal A, Mi H, Karlak B, Daverman R, et al. PANTHER: a library of protein families and subfamilies indexed by function. Genome Res. 2003;13(9):2129-41. https://doi.org/10.1101/gr.77240 3.

24. Ashwal-Fluss R, Meyer M, Pamudurti NR, Ivanov A, Bartok O, Hanan M, et al. circRNA biogenesis competes with pre-mRNA splicing. Mol Cell. 2014;56(1):55-66. https://doi.org/10.1016/j.molcel.2014.08.019.

25. Ragan C, Goodall GJ, Shirokikh NE, Preiss T. Insights into the biogenesis and potential functions of exonic circular RNA. Sci Rep. 2019;9(1):2048. https://doi.org/10.1038/s41598-018-37037-0.

26. Xie L, Mao M, Xiong K, Jiang B. Circular RNAs: a novel player in development and disease of the central nervous system. Front Cell Neurosci. 2017;11:354. https://doi.org/10.3389/fncel.2017.00354. 
27. Rybak-Wolf A, Stottmeister C, Glažar P, Jens M, Pino N, Giusti S, et al. Circular RNAs in the mammalian brain are highly abundant, conserved, and dynamically expressed. Mol Cell. 2015;58(5):870-85. https://doi. org/10.1016/j.molcel.2015.03.027.

28. Hanan M, Soreq H, Kadener S. CircRNAs in the brain. RNA Biol. 2017;14(8):1028-34. https://doi.org/10.1080/15476286.2016.1255398.

29. I parraguirre L, Muñoz-Culla M, Prada-Luengo I, Castillo-Triviño T, Olascoaga J, Otaegui D. Circular RNA profiling reveals that circular RNAs from ANXA2 can be used as new biomarkers for multiple sclerosis. Hum Mol Genet. 2017;26(18):3564-72. https://doi.org/10.1093/hmg/dd×243.

30. Gong GH, An FM, Wang Y, Bian M, Wang D, Wei CX. Comprehensive circular RNA profiling reveals the regulatory role of the CircRNA-0067835/ miR-155 pathway in temporal lobe epilepsy. Cell Physiol Biochem. 2018;51(3):1399-409. https://doi.org/10.1159/000495589.

31. Ojha R, Nandani R, Chatterjee N, Prajapati VK. Emerging role of circular RNAs as potential biomarkers for the diagnosis of human diseases. Adv Exp Med Biol. 2018;1087:141-57. https://doi. org/10.1007/978-981-13-1426-1_12.

32. Mehta SL, Pandi G, Vemuganti R. Circular RNA expression profiles alter significantly in mouse brain after transient focal ischemia. Stroke. 2017;48(9):2541-8. https://doi.org/10.1161/strokeaha.117.017469.

33. Liu C, Zhang C, Yang J, Geng X, Du H, Ji X, et al. Screening circular RNA expression patterns following focal cerebral ischemia in mice. Oncotarget. 2017;8(49):86535-47. https://doi.org/10.18632/oncotarget.21238.

34. Fonseca AC, Alves P, Inácio N, Marto JP, Viana-Baptista M, Pinho-E-Melo $T$, et al. Patients with undetermined stroke have increased atrial fibrosis: a cardiac magnetic resonance imaging study. Stroke. 2018;49(3):734-7. https://doi.org/10.1161/strokeaha.117.019641.

35. Bai $Y$, Zhang $Y$, Han B, Yang L, Chen X, Huang R, et al. Circular RNA DLGAP4 ameliorates ischemic stroke outcomes by targeting miR-143 to regulate endothelial-mesenchymal transition associated with blood-brain barrier integrity. J Neurosci. 2018;38(1):32-50. https://doi.org/10.1523/jneur osci.1348-17.2017.

36. Han B, Zhang Y, Bai Y, Chen X, Huang R, Wu F, et al. Novel insight into circular RNA HECTD1 in astrocyte activation via autophagy by targeting MIR142-TIPARP: implications for cerebral ischemic stroke. Autophagy. 2018;14(7):1164-84. https://doi.org/10.1080/15548627.2018.1458173.

37. Peng $X$, Jing $P$, Chen J, Xu L. The role of circular RNA HECTD1 expression in disease risk, disease severity, inflammation, and recurrence of acute ischemic stroke. J Clin Lab Anal. 2019. https://doi.org/10.1002/jcla.22954.

38. Wu F, Han B, Wu S, Yang L, Leng S, Li M, et al. Circular RNA TLK1 aggravates neuronal injury and neurological deficits after ischemic stroke via miR-335-3p/TIPARP. J Neurosci. 2019. https://doi.org/10.1523/jneur osci.0299-19.2019.

39. Seo WK, Jung JM, Kim JH, Koh SB, Bang OY, Oh K. Free fatty acid is associated with thrombogenicity in cardioembolic stroke. Cerebrovasc Dis. 2017:44(3-4):160-8. https://doi.org/10.1159/000478895.

40. Seo WK, Kim J, Kim YH, Kim JH, Oh K, Koh SB, et al. Elevated free fatty acid is associated with cardioembolic stroke subtype. Can J Neurol Sci. 2011;38(6):874-9.

41. Choi JY, Jung JM, Kwon DY, Park MH, Kim JH, Oh K, et al. Free fatty acid as an outcome predictor of atrial fibrillation-associated stroke. Ann Neurol. 2016;79(2):317-25. https://doi.org/10.1002/ana.24568.
42. Cho KI, Kim BJ, Cho SH, Lee JH, Kim MK, Yoo BG. Epicardial fat thickness and free fatty acid level are predictors of acute ischemic stroke with atrial fibrillation. J Cardiovasc Imag. 2018;26(2):65-74. https://doi.org/10.4250/ jcvi.2018.26.e1.

43. Golaszewska K, Harasim-Symbor E, Polak-Iwaniuk A, Chabowski A. Serum fatty acid binding proteins as a potential biomarker in atrial fibrillation. J Physiol Pharmacol. 2019. https://doi.org/10.26402/jpp.2019.1.11.

44. Hoppel C. The role of carnitine in normal and altered fatty acid metabolism. Am J Kidney Dis. 2003:41 (4 Suppl 4):S4-12.

45. Hallen A, Jamie JF, Cooper AJ. Lysine metabolism in mammalian brain: an update on the importance of recent discoveries. Amino Acids. 2013;45(6):1249-72. https://doi.org/10.1007/s00726-013-1590-1.

46. Guo X, Li Z, Zhou Y, Yu S, Yang H, Zheng L, et al. Metabolic profile for prediction of ischemic stroke in chinese hypertensive population. J Stroke Cerebrovasc Dis. 2019;28(4):1062-9. https://doi.org/10.1016/j.jstrokecer ebrovasdis.2018.12.035.

47. Lee Y, Khan A, Hong S, Jee SH, Park YH. A metabolomic study on high-risk stroke patients determines low levels of serum lysine metabolites: a retrospective cohort study. Mol BioSyst. 2017;13(6):1109-20. https://doi. org/10.1039/c6mb00732e.

48. HeW, Wei D, Cai D, Chen S, Li S, Chen W. Altered long non-coding rna transcriptomic profiles in ischemic stroke. Hum Gene Ther. 2018;29(6):719-32. https://doi.org/10.1089/hum.2017.064.

49. Maron BJ, Maron MS. Hypertrophic cardiomyopathy. Lancet. 2013;381 (9862):242-55. https://doi.org/10.1016/s0140-6736(12)60397-3.

50. Zang J, Lu D, Xu A. The interaction of circRNAs and RNA binding proteins: an important part of circRNA maintenance and function. J Neurosci Res. 2018. https://doi.org/10.1002/jnr.24356.

51. Wu C, So J, Davis-Dusenbery BN, Qi HH, Bloch DB, Shi Y, et al. Hypoxia potentiates microRNA-mediated gene silencing through posttranslational modification of Argonaute2. Mol Cell Biol. 2011;31(23):4760-74. https://doi.org/10.1128/mcb.05776-11.

52. Liu XS, Fan BY, Pan WL, Li C, Levin AM, Wang X, et al. Identification of miRNomes associated with adult neurogenesis after stroke using Argonaute 2-based RNA sequencing. RNA Biol. 2017;14(5):488-99. https://doi. org/10.1080/15476286.2016.1196320.

53. Baechtold H, Kuroda M, Sok J, Ron D, Lopez BS, Akhmedov AT. Human 75-kDa DNA-pairing protein is identical to the pro-oncoprotein TLS/FUS and is able to promote D-loop formation. J Biol Chem. 1999;274(48):34337-42. https://doi.org/10.1074/jbc.274.48.34337.

54. Zhou Y, Liu S, Liu G, Oztürk A, Hicks GG. ALS-associated FUS mutations result in compromised FUS alternative splicing and autoregulation. PLoS Genet. 2013;9(10):e1003895. https://doi.org/10.1371/journal.pgen.10038 95.

\section{Publisher's Note}

Springer Nature remains neutral with regard to jurisdictional claims in published maps and institutional affiliations.

Ready to submit your research? Choose BMC and benefit from:

- fast, convenient online submission

- thorough peer review by experienced researchers in your field

- rapid publication on acceptance

- support for research data, including large and complex data types

- gold Open Access which fosters wider collaboration and increased citations

- maximum visibility for your research: over 100M website views per year

At BMC, research is always in progress.

Learn more biomedcentral.com/submissions 